\title{
LOG IMPROVEMENT OF THE PRODI-SERRIN CRITERIA FOR NAVIER-STOKES EQUATIONS*
}

\author{
CHI HIN $\mathrm{CHAN}^{\dagger}$ AND ALEXIS VASSEUR ${ }^{\dagger}$
}

\begin{abstract}
This article is devoted to a Log improvement of Prodi-Serrin criterion for global regularity to solutions to Navier-Stokes equations in dimension 3. It is shown that the global regularity holds under the condition that $|u|^{5} /(\log (1+|u|))$ is integrable in space time variables.
\end{abstract}

Key words. Navier-Stokes, regularity criterion, a priori estimates

AMS subject classifications. 35B65, 76D03, 76D05

1. Introduction. In this article, we consider the Navier-Stokes equation on $\mathbb{R}^{3}$, given by

$$
\begin{gathered}
\partial_{t} u-\triangle u+\operatorname{div}(u \otimes u)+\nabla p=0, \\
\operatorname{div}(u)=0,
\end{gathered}
$$

where $u$ is a vector-valued function representing the velocity of the fluid, and $p$ is the pressure. Note that the pressure depends in a non local way on the velocity $u$. It can be seen as a Lagrange multiplier associated to the incompressible condition (2). The initial value problem of the above equation is endowed with the condition that $u(0, \cdot)=u_{0} \in L^{2}\left(\mathbb{R}^{3}\right)$. Leray [15] and Hopf [10] had already established the existence of global weak solutions for the Navier-Stokes equation. In particular, Leray introduced a notion of weak solutions for the Navier-Stokes equation, and proved that, for every given initial datum $u_{0} \in L^{2}\left(\mathbb{R}^{3}\right)$, there exists a global weak solution $u \in L^{\infty}\left(0, \infty ; L^{2}\left(\mathbb{R}^{3}\right)\right) \cap L^{2}\left(0, \infty ; \dot{H}^{1}\left(\mathbb{R}^{3}\right)\right)$ verifying the Navier-Stokes equation in the sense of distribution. From that time on, much effort has been devoted to establish the global existence and uniqueness of smooth solutions to the Navier-Stokes equation. Different Criteria for regularity of the weak solutions have been proposed. The ProdiSerrin conditions (see Serrin [20], Prodi [18], and [21]) states that any weak Leray-Hopf solution verifying $u \in L^{p}\left(0, \infty ; L^{q}\left(\mathbb{R}^{3}\right)\right)$ with $2 / p+3 / q=1,2 \leq p<\infty$, is regular on $(0, \infty) \times \mathbb{R}^{3}$. Notice that if $p=q$, this corresponds to $u \in L^{5}\left((0, \infty) \times \mathbb{R}^{3}\right)$. The limit case of $L^{\infty}\left(0, \infty ; L^{3}\left(\mathbb{R}^{3}\right)\right)$ has been solved very recently by L. Escauriaza, G. Seregin, and V. Sverak (see [11]). Other criterions have been later introduced, dealing with some derivatives of the velocity. Beale Kato and Majda [1] showed the global regularity under the condition that the vorticity $\omega=\operatorname{curl} u$ lies in $L^{\infty}\left(0, \infty ; L^{1}\left(\mathbb{R}^{3}\right)\right.$ ) (see Kozono and Taniuchi for improvement of this result [13]). Beirão da Veiga show in [2] that the boundedness of $\nabla u$ in $L^{p}\left(0, \infty ; L^{q}\left(\mathbb{R}^{3}\right)\right)$ for $2 / p+3 / q=2,1<p<\infty$ ensures the global regularity. In [7], Constantin and Fefferman gave a condition involving only the direction of the vorticity. Improvements of this results were obtained by Beirão da Veiga $[3,4,5,6]$, and Zhou [24, 25]. Let us also cite a condition involving the lower bound of the pressure introduced by Seregin and Sverak in [19], and conditions involving only one of the component of $u$ (see Penel and Pokorny [17], He [9], and Zhou $[23,26])$.

\footnotetext{
*Received May 15, 2007; accepted for publication May 20, 2008.

${ }^{\dagger}$ Department of Mathematics, University of Texas at Austin, Austin, TX 78712-0257, USA (cchan@math.utexas.edu; vasseur@math.utexas.edu).
} 
This article is devoted to the following log improvement of the Prodi-Serrin criterion corresponding to $p=q=5$ :

Theorem 1. Suppose that $u$ is a weak Leray-Hopf solution of the Navier-Stokes equation (1) (2) satisfying

$$
\int_{0}^{\infty} \int_{\mathbb{R}^{3}} \frac{|u|^{5}}{\log (1+|u|)} d x d s<\infty
$$

then, $u \in C^{\infty}\left((0, \infty) \times \mathbb{R}^{3}\right)$.

Montgomery-Smith introduced the following criterium in [16]:

$$
\int_{0}^{\infty} \frac{\|u(t)\|_{L^{q}\left(\mathbb{R}^{3}\right)}^{p}}{1+\log ^{+}\|u(t)\|_{L^{q}\left(\mathbb{R}^{3}\right)}} d t<\infty .
$$

Notice that the log improvement is, here, in time only. This can be seen as a natural Gronwall type extension of the Prodi-Serrin conditions. So we can see it as a one dimension ODE type extension.

The goal of our result is to extend this log improvement also in $x$. For this purpose we focused on the homogeneous case $p=q=5$, even though extension to the Prodi-Serrin range $2 \leq p<\infty$ should be doable.

The proof of Theorem 1 is split into two parts. The first point is to show that for any time $t>\lambda$, the $L^{\infty}$ norm of $u$ in $x$ can be bounded in a affine way by

$$
\int_{0}^{t} \int_{\mathbb{R}^{3}}|u|^{6} d x d t
$$

More precisely, we will show the following Proposition:

Proposition 1.1. For every $\lambda$ satisfying $0<\lambda<2$, there exists some universal constant $A_{\lambda}>0$, depending only on $\lambda$, such that, for any solution $u$ of the NavierStokes equation on $(0, \infty) \times \mathbb{R}^{3}$, we have $\|u(T, \cdot)\|_{L^{\infty}\left(\mathbb{R}^{3}\right)} \leqslant A_{\lambda}\left\{1+\int_{0}^{T} \int_{\mathbb{R}^{3}}|u|^{6} d x d s\right\}$, for any $T>\lambda$.

Then Theorem 1 follows from a Gronwall argument on $\|u(t)\|_{L^{\infty}\left(\mathbb{R}^{3}\right)}$, since:

$$
\begin{gathered}
\|u(t)\|_{L^{\infty}\left(\mathbb{R}^{3}\right)} \leq A_{\lambda} \\
+A_{\lambda} \int_{\lambda}^{t}\|u(s)\|_{L^{\infty}\left(\mathbb{R}^{3}\right)} \log \left(1+\|u(s)\|_{L^{\infty}\left(\mathbb{R}^{3}\right)}\right)\left(\int_{\mathbb{R}^{3}} \frac{|u(s)|^{5}}{\log (1+|u(s)|)} d x\right) d s
\end{gathered}
$$

and the Hypothesis gives that $\int_{\mathbb{R}^{3}} \frac{|u(s)|^{5}}{\log (1+|u(s)|)} d x$ lies in $L^{1}(0, \infty)$.

Notice that the inequality of Proposition 1.1 needs to be invariant by the scaling of the Navier-Stokes equation:

$$
u_{\varepsilon}(t, x)=\varepsilon u\left(t_{0}+\varepsilon^{2} t, x_{0}+\varepsilon x\right) .
$$

This is why the $L^{6}$ norm pops up, since it has the same scaling as that of the $L^{\infty}$ norm. Taking advantage of the scaling (3), Proposition 1.1 will follow from the following rescaled Proposition:

Proposition 1.2. There exists a universal positive constant $C^{*}$, such that for any solution $u$ of the Navier-Stokes equation on $[-1,1] \times \mathbb{R}^{3}$ satisfying $\|u\|_{L^{6}\left(\mathbb{R}^{3} \times[-1,1]\right)} \leqslant C^{*}$, we have $|u| \leqslant 1$ almost everywhere on $\left[-\frac{1}{2}, 1\right] \times \mathbb{R}^{3}$. 
The proof of proposition 1.2 is in the same spirit as the proof given by A. Vasseur [22]. It relies on a method first introduced by De Giorgi to show regularity of solutions to elliptic equations with rough diffusion coefficients [8]. In this paper, the proof of proposition 1.2 is established though sections $2,3,4$ and 5 . In section 6 , we will deduce proposition 1.1 from proposition 1.2. Finally, in the last section of this paper, we will use the conclusion of proposition 1.1, together with the fundamental result of Serrin [20], to obtain the result of Theorem 1.

2. Basic setting of the whole paper. In order to prove proposition 1.2 , we would like to introduce some notation first. Then, we will state two lemmas and one proposition which are related to the proof of proposition 1.2. So, let us fix our notation as follow.

- for each $k \geqslant 0$, let $Q_{k}=\left[T_{k}, 1\right] \times \mathbb{R}^{3}$, in which $T_{k}=-\frac{1}{2}\left(1+\frac{1}{2^{k}}\right)$.

- for each $k \geqslant 0$, let $v_{k}=\left\{|u|-\left(1-\frac{1}{2^{k}}\right)\right\}_{+}$.

- for each $k \geqslant 0$, let $d_{k}^{2}=\left.\frac{\left(1-\frac{1}{2^{k}}\right)}{|u|} \chi_{\left\{|u|>\left(1-\frac{1}{2^{k}}\right)\right\}}|\nabla| u\right|^{2}+\frac{v_{k}}{|u|}|\nabla u|^{2}$.

- for each $k \geqslant 0$, let $U_{k}=\frac{1}{2}\left\|v_{k}\right\|_{L^{\infty}\left(T_{k}, 1 ; L^{2}\left(\mathbb{R}^{3}\right)\right)}^{2}+\int_{T_{k}}^{1} \int_{\mathbb{R}^{3}} d_{k}^{2} d x d t$.

With the above setting, we are now ready to state the lemmas and proposition which are related to proposition 1.2 as follow.

LEMMA 2.1. For any solution $u$ of the Navier-Stokes equation on $[-1,1] \times \mathbb{R}^{3}$ satisfying $\|u\|_{L^{6}\left(Q_{0}\right)} \leqslant 1$, we have $U_{1} \leqslant A\|u\|_{L^{6}\left(Q_{0}\right)}^{6}$, in which $A$ is some universal constant strictly greater than 1.

Proposition 2.1. There exists some universal constants $B, \beta>1$, such that for any solution $u$ of the Navier-Stokes equation on $[-1,1] \times \mathbb{R}^{3}$ satisfying $\|u\|_{L^{6}\left(Q_{0}\right)} \leqslant$ $\frac{1}{A^{\frac{1}{6}}}$, we have $U_{k} \leqslant B^{k} U_{k-1}^{\beta}$, for all $k \geqslant 1$. Here, $A$ is the universal constant appearing in Lemma 2.1 .

Let us first show that Lemma 2.1 and Proposition 2.1 provide the result of Proposition 1.2.First we show that the sequence $U_{k}$ converges to 0 when $k$ goes to infinity. We can use for instance the following easy lemma (see [22]):

LEMMA 2.2. For any given constants $B, \beta>1$, there exists some constant $C_{0}^{*}$ such that for any sequence $\left\{a_{k}\right\}_{k \geqslant 1}$ satisfying $0<a_{1} \leq C_{0}^{*}$ and $a_{k} \leqslant B^{k} a_{k-1}^{\beta}$, for any $k \geqslant 1$, we have $\lim _{k \rightarrow \infty} a_{k}=0$.

Indeed, let $B, \beta>1$ be the constants occurring in proposition 2.1 , and let $C_{0}^{*}$ be the constant associated to $B, \beta$ in the sense of lemma 2.2. Now, take $C^{*}=$ $\min \left\{\frac{1}{A^{\frac{1}{6}}},\left(\frac{C_{0}^{*}}{A}\right)^{\frac{1}{6}}\right\}$, in which $A$ is the universal constant appearing in Lemma 2.1. Then, for any solution $u$ of the Navier-Stokes system on $[-1,1] \times \mathbb{R}^{3}$ satisfying $\|u\|_{L^{6}\left(Q_{0}\right)} \leqslant$ $C^{*}$, we have $\|u\|_{L^{6}\left(Q_{0}\right)} \leqslant\left(\frac{1}{A}\right)^{\frac{1}{6}}$. Hence, proposition 2.1 tells us that $U_{k} \leqslant B^{k} U_{k-1}^{\beta}$, for all $k \geqslant 1$ must be valid. On the other hand, Since $\|u\|_{L^{6}\left(Q_{0}\right)} \leqslant C^{*} \leqslant 1$, Lemma 2.1 also implies that $U_{1} \leqslant A\|u\|_{L^{6}\left(Q_{0}\right)}^{6} \leqslant C_{0}^{*}$. Hence, it follows from Lemma 2.2 that $\lim _{k \rightarrow \infty} U_{k}=0$. However, since we have the inequality

$$
\frac{1}{2} \int_{\mathbb{R}^{3}}\{|u(t, x)|-1\}_{+}^{2} d x \leqslant \frac{1}{2} \sup _{t \in\left[-\frac{1}{2}, 1\right]} \int_{\mathbb{R}^{3}} v_{k}^{2} d x \leqslant U_{k},
$$

for every $t \in\left[-\frac{1}{2}, 1\right]$. As a result, $\lim _{k \rightarrow \infty} U_{k}=0$ immediately implies that $|u| \leqslant 1$ almost everywhere on $\left[-\frac{1}{2}, 1\right] \times \mathbb{R}^{3}$. This gives the result of Proposition 1.2. 
3. Proof of Lemma 2.1. In this section, we will devote our effort in proving Lemma 2.1. Let us recall that the Navier-Stokes equation on $(-\infty, \infty) \times \mathbb{R}^{3}$ is

$$
\partial_{t} u-\triangle u+\operatorname{div}(u \otimes u)+\nabla P=0,
$$

together with the divergence free condition $\operatorname{div}(u)=0$. Now, by multiplying the above equation by the term $\frac{v_{1}}{|u|} u$, we yield the following inequality, which is valid in the sense of distribution.

$$
\partial_{t}\left(\frac{1}{2} v_{1}^{2}\right)+d_{1}^{2}-\triangle\left(\frac{1}{2} v_{1}^{2}\right)+\operatorname{div}\left(\frac{v_{1}^{2}}{2} u\right)+\frac{v_{1}}{|u|} u \nabla P \leqslant 0 .
$$

Consider now the variables $\sigma, t$ with $T_{0} \leqslant \sigma \leqslant T_{1} \leqslant t \leqslant 1$, where $T_{0}=-1$, and $T_{1}=-\frac{1}{2}\left(1+\frac{1}{2}\right)$. We mention that we have the following, which is valid in the sense of distribution.

- $\int_{\sigma}^{t} \int_{\mathbb{R}^{3}} \partial_{t}\left(\frac{1}{2} v_{1}^{2}\right) d x d s=\frac{1}{2} \int_{\mathbb{R}^{3}} v_{1}^{2}(t, x) d x-\frac{1}{2} \int_{\mathbb{R}^{3}} v_{1}^{2}(\sigma, x) d x$.

- $\int_{\sigma}^{t} \int_{\mathbb{R}^{3}} \operatorname{div}\left(\frac{v_{1}^{2}}{2} u\right)-\triangle\left(\frac{v_{1}^{2}}{2}\right) d x d s=0$.

Hence, by taking the integral over $[\sigma, t] \times \mathbb{R}^{3}$ to the above inequality, we yield the following estimation.

$$
\begin{aligned}
\frac{1}{2} \int_{\mathbb{R}^{3}} v_{1}^{2}(t, x) d x+\int_{\sigma}^{t} \int_{\mathbb{R}^{3}} d_{k}^{2} d x d s & \leqslant \frac{1}{2} \int_{\mathbb{R}^{3}} v_{1}^{2}(\sigma, x) d x+\int_{\sigma}^{t}\left|\int_{\mathbb{R}^{3}} \frac{v_{1}}{|u|} u \nabla P d x\right| d s \\
& =\frac{1}{2} \int_{\mathbb{R}^{3}} v_{1}^{2}(\sigma, x) d x+\int_{\sigma}^{t}\left|\int_{\mathbb{R}^{3}} P \nabla\left(\frac{v_{1}}{|u|} u\right) d x\right| d s \\
& \leqslant \frac{1}{2} \int_{\mathbb{R}^{3}} v_{1}^{2}(\sigma, x) d x+3 \int_{\sigma}^{t} \int_{\mathbb{R}^{3}} d_{1}|P| \chi_{\left\{v_{1}>0\right\}} d x d s \\
& \leqslant \frac{1}{2} \int_{\mathbb{R}^{3}} v_{1}^{2}(\sigma, x) d x+\frac{3}{2} \int_{\sigma}^{t} \int_{\mathbb{R}^{3}} \alpha^{2} d_{1}^{2} d x d s \\
& +\frac{3}{2} \int_{\sigma}^{t} \int_{\mathbb{R}^{3}} \frac{|P|^{2}}{\alpha^{2}} \chi_{\left\{v_{1}>0\right\}} d x d s,
\end{aligned}
$$

in which $\alpha$ can be any positive constant (In the third step of the above deduction, we have used the nontrival fact that $\left|\nabla\left(\frac{v_{k}}{|u|} u\right)\right| \leqslant 3 d_{k}$, whose justification will be given in the last part of Section 4). Hence we yield the following inequality which is valid for any $\alpha>0$.

$$
\begin{aligned}
\int_{\mathbb{R}^{3}} \frac{v_{1}^{2}(t, x)}{2} d x+\int_{\sigma}^{t} \int_{\mathbb{R}^{3}} \frac{\left(2-3 \alpha^{2}\right) d_{1}^{2}}{2} d x d s & \leqslant \int_{\mathbb{R}^{3}} \frac{v_{1}^{2}(\sigma, x)}{2} d x \\
& +\int_{\sigma}^{t} \int_{\mathbb{R}^{3}} \frac{3|P|^{2} \chi_{\left\{v_{1}>0\right\}}}{2 \alpha^{2}} d x d s .
\end{aligned}
$$

If we choose $\alpha=\left(\frac{1}{2}\right)^{\frac{1}{2}}$, then the inequality shown as above becomes

$$
\frac{1}{2} \int_{\mathbb{R}^{3}} v_{1}^{2}(t, x) d x+\frac{1}{4} \int_{\sigma}^{t} \int_{\mathbb{R}^{3}} d_{1}^{2} d x d s \leqslant \int_{\mathbb{R}^{3}} \frac{v_{1}^{2}(\sigma, x)}{2} d x+3 \int_{\sigma}^{t} \int_{\mathbb{R}^{3}}|P|^{2} \chi_{\left\{v_{1}>0\right\}} d x d s .
$$

By taking average over $\sigma \in\left[T_{0}, T_{1}\right]$, we can carry out the following estiamtion

$$
\int_{\mathbb{R}^{3}} \frac{v_{1}^{2}(t, x)}{2} d x+\int_{T_{1}}^{t} \int_{\mathbb{R}^{3}} \frac{d_{1}^{2}}{4} d x d s \leqslant \frac{4}{2} \int_{-1}^{T_{1}} \int_{\mathbb{R}^{3}} v_{1}^{2}(\sigma, x)+3 \int_{-1}^{t} \int_{\mathbb{R}^{3}}|P|^{2} \chi_{\left\{v_{1}>0\right\}} d x d s .
$$


Notice that, in the above inequality, the integer 4 appears in the first term of the right hand side because $\frac{1}{T_{k}-T_{k-1}}=2^{k+1}$. Now, by taking the $L^{\infty}$-norm over $t \in\left[T_{1}, 1\right]$, we yield

$$
\frac{1}{4} U_{1} \leqslant 2 \int_{-1}^{T_{1}} \int_{\mathbb{R}^{3}} v_{1}^{2} d x d s+3 \int_{Q_{0}}|P|^{2} \chi_{\left\{v_{1}>0\right\}} .
$$

But, we notice that

$$
\begin{aligned}
\int_{-1}^{T_{1}} \int_{\mathbb{R}^{3}} v_{1}^{2} d x d s \leqslant \int_{Q_{0}} v_{1}^{2} \chi_{\left\{v_{1}>0\right\}} & \leqslant\left(\int_{Q_{0}} v_{1}^{6}\right)^{\frac{1}{3}}\left(\int_{Q_{0}} \chi_{\left\{v_{1>0}\right\}}\right)^{\frac{2}{3}} \\
& \leqslant\|u\|_{L^{6}\left(Q_{0}\right)}^{2}\left(\int_{Q_{0}} \chi_{\left\{v_{0}>\frac{1}{2}\right\}}\right)^{\frac{2}{3}} \\
& \leqslant\|u\|_{L^{6}\left(Q_{0}\right)}^{2}\left(2^{6} \int_{Q_{0}} v_{0}^{6}\right)^{\frac{2}{3}}=2^{4}\|u\|_{L^{6}\left(Q_{0}\right)}^{6} .
\end{aligned}
$$

On the other hand, since the pressure $P$ satisfies the equation $-\triangle P=$ $\sum \partial_{i} \partial_{j}\left(u_{i} u_{j}\right)$.

So, by the Riesz theorem in the theory of singular integral, we have $\|P\|_{L^{3}\left(Q_{0}\right)} \leqslant$ $C_{3}\|u\|_{L^{6}\left(Q_{0}\right)}^{2}$, in which $C_{3}$ is some universal constant . Hence, it follows that

$$
\begin{aligned}
\int_{Q_{0}}|P|^{2} \chi_{\left\{v_{1}>0\right\}} \leqslant\|P\|_{L^{3}\left(Q_{0}\right)}^{2}\left\|\chi_{\left\{v_{1}>0\right\}}\right\|_{L^{3}\left(Q_{0}\right)} & \leqslant C_{3}^{2}\|u\|_{L^{6}\left(Q_{0}\right)}^{4}\left\|\chi_{\left\{v_{0}>\frac{1}{2}\right\}}\right\|_{L^{3}\left(Q_{0}\right)} \\
& \leqslant C_{3}^{2}\|u\|_{L^{6}\left(Q_{0}\right)}^{4}\left(2^{6} \int_{Q_{0}} v_{0}^{6}\right)^{\frac{1}{3}} \\
& =4 C_{3}^{2}\|u\|_{L^{6}\left(Q_{0}\right)}^{6} .
\end{aligned}
$$

Hence it follows that

$$
\frac{1}{4} U_{1} \leqslant 2 \int_{Q_{0}} v_{1}^{2}+3 \int_{Q_{0}}|P|^{2} \chi_{\left\{v_{1}>0\right\}} \leqslant 2^{5}\|u\|_{L^{6}\left(Q_{0}\right)}^{6}+12 C_{3}^{2}\|u\|_{L^{6}\left(Q_{0}\right)}^{6} .
$$

As a result, by taking $A=2^{7}+48 C_{3}^{2}$, we can at once deduce that

$$
U_{1} \leqslant A\|u\|_{L^{6}\left(Q_{0}\right)}^{6} .
$$

So, we are done in establishing Lemma 2.1

\section{Preliminaries for the proof of Proposition 2.1.}

Lemma 4.1. There exists some constant $C>0$, such that for any $k \geqslant 1$, and any $F \in L^{\infty}\left(T_{k}, 1 ; L^{2}\left(\mathbb{R}^{3}\right)\right)$ with $\nabla F \in L^{2}\left(Q_{k}\right)$, we have $\|F\|_{L^{\frac{10}{3}}\left(Q_{k}\right)} \leqslant$ $C\|F\|_{L^{\infty}\left(T_{k}, 1 ; L^{2}\left(\mathbb{R}^{3}\right)\right)}^{\frac{2}{5}}\|\nabla F\|_{L^{2}\left(Q_{k}\right)}^{\frac{3}{5}}$.

Proof. By Sobolev-embedding Theorem, there is a constant $C$, depending only on the dimension of $\mathbb{R}^{3}$, such that

$$
\left(\int_{\mathbb{R}^{3}}|F(t, x)|^{6} d x\right)^{\frac{1}{6}} \leqslant C\left(\int_{\mathbb{R}^{3}}|\nabla F(t, x)|^{2} d x\right)^{\frac{1}{2}} .
$$


For any $t \in\left[T_{k}, 1\right]$, where $k \geqslant 1$, and $F$ is some function which verifies $F \in$ $L^{\infty}\left(T_{k}, 1 ; L^{2}\left(\mathbb{R}^{3}\right)\right)$, and $\nabla F \in L^{2}\left(Q_{k}\right)$. By taking the power 2 on both sides of the above inequality and then taking integration along the variable $t \in\left[T_{k}, 1\right]$, we yield

$$
\int_{T_{k}}^{1}\left(\int_{\mathbb{R}^{3}}|F|^{6} d x\right)^{\frac{1}{3}} d t \leqslant C^{2} \int_{T_{k}}^{1} \int_{\mathbb{R}^{3}}|\nabla F|^{2} d x d t .
$$

On the other hand, by Holder's inequality, we have

$$
\begin{aligned}
\|F\|_{L^{\frac{10}{3}}\left(Q_{k}\right)}^{\frac{10}{3}}=\int_{T_{k}}^{1} \int_{\mathbb{R}^{3}}|F|^{2}|F|^{\frac{4}{3}} d x d t & \leqslant \int_{T_{k}}^{1}\left(\int_{\mathbb{R}^{3}}|F|^{6} d x\right)^{\frac{1}{3}}\left(\int_{\mathbb{R}^{3}}|F|^{2} d x\right)^{\frac{2}{3}} d t \\
& \leqslant\|F\|_{L^{\infty}\left(T_{k}, 1 ; L^{2}\left(\mathbb{R}^{3}\right)\right)}^{\frac{4}{3}}\|F\|_{L^{2}\left(T_{k}, 1 ; L^{6}\left(\mathbb{R}^{3}\right)\right)}^{2}
\end{aligned}
$$

By taking the advantage that $\|F\|_{L^{2}\left(T_{k}, 1 ; L^{6}\left(\mathbb{R}^{3}\right)\right)} \leqslant C\|\nabla F\|_{L^{2}\left(Q_{k}\right)}$, we yield

$$
\|F\|_{L^{\frac{10}{3}}\left(Q_{k}\right)}^{\frac{10}{3}} \leqslant C^{2}\|F\|_{L^{\infty}\left(T_{k}, 1 ; L^{2}\left(\mathbb{R}^{3}\right)\right)}^{\frac{4}{3}}\|\nabla F\|_{L^{2}\left(Q_{k}\right)}^{2} .
$$

Hence, we have

$$
\|F\|_{L^{\frac{10}{3}}\left(Q_{k}\right)} \leqslant C\|F\|_{L^{\infty}\left(T_{k}, 1 ; L^{2}\left(\mathbb{R}^{3}\right)\right)}^{\frac{2}{5}}\|\nabla F\|_{L^{2}\left(Q_{k}\right)}^{\frac{3}{5}} .
$$

so, we are done. $\square$

Lemma 4.2. For any $1<q<\infty$, we have $\left\|\chi_{\left\{v_{k}>0\right\}}\right\|_{L^{q}\left(Q_{k-1}\right)} \leqslant 2^{\frac{10 k}{3 q}} C^{\frac{1}{q}} U_{k-1}^{\frac{5}{3 q}}$.

Proof. First, we have to notice that $\left\{v_{k}>0\right\}$ is a subset of $\left\{v_{k-1}>\frac{1}{2^{k}}\right\}$, hence we have

$$
\int_{Q_{k-1}} \chi_{\left\{v_{k}>0\right\}} \leqslant \int_{Q_{k-1}} \chi_{\left\{v_{k-1}>\frac{1}{2^{k}}\right\}} \leqslant 2^{\frac{10 k}{3}} \int_{Q_{k-1}}\left|v_{k-1}\right|^{\frac{10}{3}} .
$$

By our previous Lemma, we have

$$
\begin{aligned}
\left\|v_{k-1}\right\|_{L^{\frac{10}{3}}}^{\frac{10}{3}}\left(Q_{k-1}\right) & \leqslant C^{2}\left\|v_{k-1}\right\|_{L^{\infty}\left(T_{k-1}, 1 ; L^{2}\left(\mathbb{R}^{3}\right)\right)}^{\frac{4}{3}}\left\|\nabla v_{k-1}\right\|_{L^{2}\left(Q_{k-1}\right)}^{2} \\
& \leqslant C^{2}\left(U_{k-1}^{\frac{1}{2}}\right)^{\frac{4}{3}}\left\|d_{k-1}\right\|_{L^{2}\left(Q_{k-1}\right)}^{2} \\
& \leqslant C^{2} U_{k-1}^{\frac{2}{3}} U_{k-1} \\
& =C^{2} U_{k-1}^{\frac{5}{3}} .
\end{aligned}
$$

So, it follows that $\int_{Q_{k-1}} \chi_{\left\{v_{k}>0\right\}} \leqslant 2^{\frac{10 k}{3}} C^{2} U_{k-1}^{\frac{5}{3}}$, and hence we have $\left\|\chi_{\left\{v_{k}>0\right\}}\right\|_{L^{q}\left(Q_{k-1}\right)} \leqslant 2^{\frac{10 k}{3 q}} C^{\frac{1}{q}} U_{k-1}^{\frac{5}{3 q}}$, where $\mathrm{C}$ is some universal constant. So, we are done. $\mathrm{C}$

In the proof of Lemma 4.2 , we have used the fact that $\left|\nabla v_{k}\right| \leqslant d_{k}$, whose justification will be given immediately in the following paragraph.

Before we leave this section, we also want to list out some inequalities which will often be used in the proof of proposition 1.1 as follow: 
- $\left|\left(1-\frac{v_{k}}{|u|}\right) u\right| \leqslant 1-\frac{1}{2^{k}}$.

- $\frac{v_{k}}{|u|}|\nabla u| \leqslant d_{k}$.

- $\chi_{\left\{v_{k}>0\right\}}|\nabla| u|| \leqslant d_{k}$.

- $\left|\nabla v_{k}\right| \leqslant d_{k}$.

- $\left|\nabla\left(\frac{v_{k}}{|u|} u\right)\right| \leqslant 3 d_{k}$.

- $d_{k} \leqslant 2^{\frac{1}{2}} d_{k-1}$, for all $k \geqslant 2$.

Now, we first want to justify the validity of $\left|\left(1-\frac{v_{k}}{|u|}\right) u\right| \leqslant 1-\frac{1}{2^{k}}$. In the case in which the point $(t, x)$ satisfies $|u(t, x)|<1-\frac{1}{2^{k}}$, we have $v_{k}(t, x)=0$, and hence it follows that

$$
\left|\left\{1-\frac{v_{k}(t, x)}{|u(t, x)|}\right\} u(t, x)\right|=|u(t, x)|<1-\frac{1}{2^{k}} .
$$

In the case in which $(t, x)$ satisfies $|u(t, x)| \geqslant 1-\frac{1}{2^{k}}$, we have $v_{k}(t, x)=|u(t, x)|-$ $\left(1-\frac{1}{2^{k}}\right)$, and hence it follows that

$$
\left|\left\{1-\frac{v_{k}}{|u|}\right\} u(t, x)\right|=\left|1-\frac{|u|-\left(1-\frac{1}{2^{k}}\right)}{|u|}\right||u|=1-\frac{1}{2^{k}} .
$$

So, no matter in which case, we always have the conclusion that $\left|\left(1-\frac{v_{k}}{|u|}\right) u\right| \leqslant$ $1-\frac{1}{2^{k}}$.

Next, according to the definition of $d_{k}^{2}$, we can carry out the following estimation

$$
d_{k}^{2} \geqslant \frac{v_{k}}{|u|}|\nabla u|^{2} \geqslant\left\{\frac{v_{k}}{|u|}|\nabla u|\right\}^{2}
$$

Hence, by taking square root, it follows at once that $d_{k} \geqslant \frac{v_{k}}{|u|}|\nabla u|$.

We now turn our attention to the inequality $\chi_{\left\{|u| \geqslant\left(1-\frac{1}{2^{k}}\right)\right\}}|\nabla| u|| \leqslant d_{k}$. To justify it, we recall that $|\nabla u| \geqslant|\nabla| u||$. Hence, it follows from the definition of $d_{k}^{2}$ that

$$
d_{k}^{2} \geqslant\left.\frac{1-\frac{1}{2^{k}}}{|u|} \chi_{\left\{|u| \geqslant 1-\frac{1}{2^{k}}\right\}}|\nabla| u\right|^{2}+\left\{1-\frac{1-\frac{1}{2^{k}}}{|u|}\right\} \chi_{\left\{|u| \geqslant 1-\frac{1}{2^{k}}\right\}}|\nabla| u||^{2} .
$$

So, by simplifying the right-hand side of the above inequality, we can deduce that $d_{k}^{2} \geqslant \chi_{\left\{|u| \geqslant 1-\frac{1}{2^{k}}\right\}}|\nabla| u||^{2}$. Hence, we have $d_{k} \geqslant \chi_{\left\{|u| \geqslant 1-\frac{1}{2^{k}}\right\}}|\nabla| u||$. In addition, since it is obvious to see that $\nabla v_{k}=\chi_{\left\{|u| \geqslant 1-\frac{1}{2^{k}}\right\}} \nabla|u|$, we also have the result that $\left|\nabla v_{k}\right| \leqslant d_{k}$. Finally, we want to justify the inequality that $\left|\nabla\left(\frac{v_{k}}{|u|} u\right)\right| \leqslant 3 d_{k}$. So, we notice that, by applying the product rule, we have

$$
\nabla\left(\frac{v_{k}}{|u|} u\right)=\nabla\left(v_{k}\right) \frac{u}{|u|}+\frac{v_{k}}{|u|} \nabla u-\frac{v_{k}}{|u|^{2}} u \nabla|u|
$$

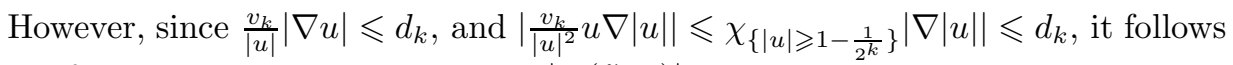
at once from the above expression that $\left|\nabla\left(\frac{v_{k}}{|u|} u\right)\right| \leqslant 3 d_{k}$.

Finally, we also need to show that $d_{k} \leqslant 2^{\frac{1}{2}} d_{k-1}$, for all $k \geqslant 2$. For this purpose, we first notice that we have the follwing inequality estimation to be valid for all $k \geqslant 2$. 


$$
\begin{aligned}
\frac{\left(1-\frac{1}{2^{k}}\right)}{|u|} \chi_{\left\{|u|>\left(1-\frac{1}{2^{k}}\right)\right\}}|\nabla| u||^{2} & \leqslant \frac{\left(1-\frac{1}{2^{k}}\right)}{|u|} \chi_{\left\{|u|>\left(1-\frac{1}{2^{k-1}}\right)\right\}}|\nabla| u||^{2} \\
& =\left.\frac{\left(1-\frac{1}{2^{k}}\right)}{\left(1-\frac{1}{2^{k-1}}\right)} \frac{\left(1-\frac{1}{2^{k-1}}\right)}{|u|} \chi_{\left\{|u|>\left(1-\frac{1}{2^{k-1}}\right)\right\}}|\nabla| u\right|^{2} \\
& \leqslant\left. 2 \frac{\left(1-\frac{1}{2^{k-1}}\right)}{|u|} \chi_{\left\{|u|>\left(1-\frac{1}{2^{k-1}}\right)\right\}}|\nabla| u\right|^{2} .
\end{aligned}
$$

We remark that the last inequality in the above estimation follows from the fact that $\frac{1}{1-\frac{1}{2^{k-1}}} \leqslant 2$, for all $k \geqslant 2$. Now, by combining the above inequality estimation with the definition of $d_{k}^{2}$, we can at once deduce that $d_{k}^{2} \leqslant 2 d_{k-1}^{2}$ is valid for all $k \geqslant 2$, and hence $d_{k} \leqslant 2^{\frac{1}{2}} d_{k-1}$, for all $k \geqslant 2$.

5. Proof of Proposition 2.1. To begin the argument, we recall that, by multiplying the equation $\partial_{t} u-\triangle u+\operatorname{div}(u \otimes u)+\nabla P=0$ on $(-\infty, \infty) \times \mathbb{R}^{3}$, we yield the following inequality formally, which is indeed valid in the sense of distribution

$$
\partial_{t}\left(\frac{v_{k}^{2}}{2}\right)+d_{k}^{2}-\triangle\left(\frac{v_{k}^{2}}{2}\right)+\operatorname{div}\left(\frac{v_{k}^{2}}{2} u\right)+\frac{v_{k}}{|u|} u \nabla P \leqslant 0
$$

Next, let us consider the variables $\sigma, t$ verifying $T_{k-1} \leqslant \sigma \leqslant T_{k} \leqslant t \leqslant 1$. Then, we have

- $\int_{\sigma}^{t} \int_{\mathbb{R}^{3}} \partial_{t}\left(\frac{v_{k}^{2}}{2}\right) d x d s=\int_{\mathbb{R}^{3}} \frac{v_{k}^{2}(t, x)}{2} d x-\int_{\mathbb{R}^{3}} \frac{v_{k}^{2}(\sigma, x)}{2} d x$.

- $\int_{\sigma}^{t} \int_{\mathbb{R}^{3}} \triangle\left(\frac{v_{k}^{2}}{2}\right) d x d s=0$.

- $\int_{\sigma}^{t} \int_{\mathbb{R}^{3}} \operatorname{div}\left(\frac{v_{k}^{2}}{2} u\right) d x d s=0$.

So, it is straightforward to see that

$$
\int_{\mathbb{R}^{3}} \frac{v_{k}^{2}(t, x)}{2} d x+\int_{\sigma}^{t} \int_{\mathbb{R}^{3}} d_{k}^{2} d x d s \leqslant \int_{\mathbb{R}^{3}} \frac{v_{k}^{2}(\sigma, x)}{2} d x+\int_{\sigma}^{t}\left|\int_{\mathbb{R}^{3}} \frac{v_{k}}{|u|} u \nabla P d x\right| d s,
$$

for any $\sigma, t$ satisfying $T_{k-1} \leqslant \sigma \leqslant T_{k} \leqslant t \leqslant 1$. By taking the average over the variable $\sigma$, we yield

$$
\int_{\mathbb{R}^{3}} \frac{v_{k}^{2}(t, x)}{2} d x+\int_{T_{k}}^{t} \int_{\mathbb{R}^{3}} d_{k}^{2} d x d s \leqslant 2^{k} \int_{T_{k-1}}^{T_{k}} \int_{\mathbb{R}^{3}} v_{k}^{2}(s, x) d x d s+\int_{T_{k-1}}^{t}\left|\int_{\mathbb{R}^{3}} \frac{v_{k}}{|u|} u \nabla P d x\right| d s
$$

By taking the sup over $t \in\left[T_{k}, 1\right]$. the above inequality will give the following

$$
U_{k} \leqslant 2^{k} \int_{Q_{k-1}} v_{k}^{2}+\int_{T_{k-1}}^{1}\left|\int_{\mathbb{R}^{3}} \frac{v_{k}}{|u|} u \nabla P d x\right| d s
$$

But, from Lemma 4.2 and Holder's inequality, we have 


$$
\begin{aligned}
\int_{Q_{k-1}} v_{k}^{2}=\int_{Q_{k-1}} v_{k}^{2} \chi_{\left\{v_{k}>0\right\}} & \leqslant\left(\int_{Q_{k-1}} v_{k}^{\frac{10}{3}}\right)^{\frac{3}{5}}\left\|\chi_{\left\{v_{k}>0\right\}}\right\|_{L^{\frac{5}{2}}\left(Q_{k-1}\right)} \\
& \leqslant\left\|v_{k}\right\|_{L^{\frac{10}{3}}}^{2}\left(Q_{k-1} 2^{\frac{4 k}{3}} C^{\frac{2}{5}} U_{k-1}^{\frac{2}{3}}\right. \\
& \leqslant\left\|v_{k-1}\right\|_{L^{\frac{10}{3}}}^{2}\left(Q_{k-1} 2^{\frac{4 k}{3}} C^{\frac{2}{5}} U_{k-1}^{\frac{2}{3}}\right. \\
& \leqslant C U_{k-1}^{\frac{5}{3}} 2^{\frac{4 k}{3}}
\end{aligned}
$$

In the above estimation, we have used the fact that $v_{k} \leqslant v_{k-1}$. As a result, we have the following conclusion

$$
U_{k} \leqslant 2^{\frac{7 k}{3}} C U_{k-1}^{\frac{5}{3}}+\int_{T_{k-1}}^{1}\left|\int_{\mathbb{R}^{3}} \frac{v_{k}}{|u|} u \nabla p d x\right| d s
$$

Now, in order to estimate the term $\int_{T_{k-1}}^{1}\left|\int_{\mathbb{R}^{3}} \frac{v_{k}}{u} u \nabla P d x\right| d s$, we would like to carry out the following computation

$$
\begin{aligned}
-\triangle P=\sum \partial_{i} \partial_{j}\left(u_{i} u_{j}\right) & =\sum \partial_{i} \partial_{j}\left\{\left(1-\frac{v_{k}}{|u|}\right) u_{i}\left(1-\frac{v_{k}}{|u|}\right) u_{j}+2\left(1-\frac{v_{k}}{|u|}\right) u_{i} \frac{v_{k}}{|u|} u_{j}\right\} \\
& +\sum \partial_{i} \partial_{j}\left\{\frac{v_{k}}{|u|} u_{i} \frac{v_{k}}{|u|} u_{j}\right\} .
\end{aligned}
$$

This motivates us to decompose $P$ as $P=P_{k 1}+P_{k 2}$, in which

$$
-\triangle P_{k 1}=\sum \partial_{i} \partial_{j}\left\{\left(1-\frac{v_{k}}{|u|}\right) u_{i}\left(1-\frac{v_{k}}{|u|}\right) u_{j}+2\left(1-\frac{v_{k}}{|u|}\right) u_{i} \frac{v_{k}}{|u|} u_{j}\right\},
$$

and that

$$
-\triangle P_{k 2}=\sum \partial_{i} \partial_{j}\left\{\frac{v_{k}}{|u|} u_{i} \frac{v_{k}}{|u|} u_{j}\right\} .
$$

First, we have to notice that:

$$
\begin{aligned}
\left|\left(1-\frac{v_{k}}{|u|}\right)^{2} u_{i} u_{j}+2\left(1-\frac{v_{k}}{|u|}\right) u_{i} \frac{v_{k}}{|u|} u_{j}\right| & \leqslant\left(1-\frac{1}{2^{k}}\right)\left\{\left(1-\frac{v_{k}}{|u|}\right)\left|u_{j}\right|+2 \frac{v_{k}}{|u|}\left|u_{j}\right|\right\} \\
& \leqslant\left(1-\frac{v_{k}}{|u|}\right)\left|u_{j}\right|+2 \frac{v_{k}}{|u|}\left|u_{j}\right| \\
& \leqslant 3\left|u_{j}\right| \leqslant 3|u| .
\end{aligned}
$$

So, by Riesz's Theorem in the theroy of singular operator, we yield

$$
\left\|P_{k 1}\right\|_{L^{6}\left(Q_{k-1}\right)} \leqslant C_{6}\|3 u\|_{L^{6}\left(Q_{k-1}\right)} \leqslant 3 C_{6}\left(\frac{1}{A}\right)^{\frac{1}{6}} \leqslant 3 C_{6} .
$$


So, we have

$$
\begin{aligned}
\int_{T_{k-1}}^{1}\left|\int_{\mathbb{R}^{3}} \frac{v_{k}}{|u|} u \nabla P_{k 1} d x\right| d s & =\int_{T_{k-1}}^{1}\left|\int_{\mathbb{R}^{3}} P_{k 1} \nabla\left(\frac{v_{k}}{|u|} u\right) d x\right| d s \\
& \leqslant 3 \int_{T_{k-1}}^{1} \int_{\mathbb{R}^{3}} d_{k}\left|P_{k 1}\right| \chi_{\left\{v_{k}>0\right\}} d x d s \\
& \leqslant 3\left\|d_{k}\right\|_{L^{2}\left(Q_{k-1}\right)}\left\|P_{k 1}\right\|_{L^{6}\left(Q_{k-1}\right)}\left\|\chi_{\left\{v_{k}>0\right\}}\right\|_{L^{3}\left(Q_{k-1}\right)} \\
& \leqslant 3\left(2^{\frac{1}{2}}\right)\left\|d_{k-1}\right\|_{L^{2}\left(Q_{k-1}\right)} 3 C_{6} 2^{\frac{10 k}{9}} C^{\frac{1}{3}} U_{k-1}^{\frac{5}{9}} \\
& \leqslant 9\left(2^{\frac{1}{2}}\right) C_{6} C^{\frac{1}{3}} U_{k-1}^{\frac{1}{2}} 2^{\frac{10 k}{9}} U_{k-1}^{\frac{5}{9}} .
\end{aligned}
$$

(In the above estimation, we have used the fact that $d_{k} \leqslant 2^{\frac{1}{2}} d_{k-1}$, for all $k \geqslant 2$ ). That is, we have the following conclusion that

$$
\int_{T_{k-1}}^{1}\left|\int_{\mathbb{R}^{3}} \frac{v_{k}}{|u|} u \nabla P_{k 1} d x\right| d s \leqslant C 2^{\frac{10 k}{9}} U_{k-1}^{\frac{19}{18}} .
$$

Next, we would like to estimate the term $\int_{T_{k-1}}^{1}\left|\int_{\mathbb{R}^{3}} \frac{v_{k}}{|u|} u \nabla P_{k 2} d x\right| d s$. First, we recall that, by the definition of $P_{k 2}$, we have

$$
P_{k 2}=\sum R_{i} R_{j}\left\{\frac{v_{k}}{|u|} u_{i} \frac{v_{k}}{|u|} u_{j}\right\},
$$

in which $R_{i}, R_{j}$ etc are the Riesz's Transforms. Hence, we have

$$
\nabla P_{k 2}=\sum R_{i} R_{j}\left\{\nabla\left(\frac{v_{k}}{|u|} u_{i} \frac{v_{k}}{|u|} u_{j}\right)\right\} .
$$

Now, we notice that

$$
\begin{aligned}
\left|\nabla\left(\frac{v_{k}}{|u|} u_{i} \frac{v_{k}}{|u|} u_{j}\right)\right| \leqslant\left|\nabla\left(\frac{v_{k}}{|u|} u_{i}\right)\right|\left|\frac{v_{k}}{|u|} u_{j}\right|+\frac{v_{k}}{|u|}\left|u_{i}\right|\left|\nabla\left(\frac{v_{k}}{|u|} u_{j}\right)\right| & \leqslant 3 d_{k} v_{k}+v_{k}\left(3 d_{k}\right) \\
& =6 v_{k} d_{k} .
\end{aligned}
$$

So, by applying the Riesz's Theorem in the theory of Singular integral, we have

$$
\begin{aligned}
\left\|\nabla P_{k 2}\right\|_{L^{\frac{3}{2}}\left(Q_{k-1}\right)} \leqslant C_{\frac{3}{2}}\left\|v_{k} d_{k}\right\|_{L^{\frac{3}{2}}\left(Q_{k-1}\right)} & \leqslant C_{\frac{3}{2}}\left\{\left(\int_{Q_{k-1}} v_{k}^{6}\right)^{\frac{1}{4}}\left(\int_{Q_{k-1}} d_{k}^{2}\right)^{\frac{3}{4}}\right\}^{\frac{2}{3}} \\
& =C_{\frac{3}{2}}\left(\int_{Q_{k-1}} v_{k}^{6}\right)^{\frac{1}{6}}\left(\int_{Q_{k-1}} d_{k}^{2}\right)^{\frac{1}{2}} \\
& \leqslant C_{\frac{3}{2}}\|u\|_{L^{6}\left(Q_{0}\right)}\left\|d_{k}\right\|_{L^{2}\left(Q_{k-1}\right)} \\
& \leqslant C_{\frac{3}{2}}\left(\frac{1}{A}\right)^{\frac{1}{6}}(2)^{\frac{1}{2}}\left\|d_{k-1}\right\|_{L^{2}\left(Q_{k-1}\right)} \\
& \leqslant 2^{\frac{1}{2}} C_{\frac{3}{2}} U_{k-1}^{\frac{1}{2}}
\end{aligned}
$$


So, by applying the generalized Holder's inequality with exponents $\frac{10}{3}, 30, \frac{3}{2}$ to the terms $v_{k}, \chi_{\left\{v_{k}>0\right\}}, \nabla P_{k 2}$ respectively, we yield

$$
\begin{aligned}
\int_{T_{k-1}}^{1}\left|\int_{\mathbb{R}^{3}} \frac{v_{k}}{|u|} u \nabla P_{k 2} d x\right| d t & \leqslant \int_{Q_{k-1}} v_{k} \chi_{\left\{v_{k}>0\right\}}\left|\nabla P_{k 2}\right| d x d t \\
& \leqslant\left\|v_{k}\right\|_{L^{\frac{10}{3}}\left(Q_{k-1}\right)}\left\|\chi_{\left\{v_{k}>0\right\}}\right\|_{L^{30}\left(Q_{k-1}\right)}\left\|\nabla P_{k 2}\right\|_{L^{\frac{3}{2}}}\left(Q_{k-1}\right) \\
& \leqslant U_{k-1}^{\frac{1}{2}} 2^{\frac{k}{9}} C^{\frac{1}{30}} U_{k-1}^{\frac{5}{90}} 2^{\frac{1}{2}} C_{\frac{3}{2}} U_{k-1}^{\frac{1}{2}} \\
& =C 2^{\frac{k}{9}} U_{k-1}^{1+\frac{5}{90}} \\
& =C 2^{\frac{k}{9}} U_{k-1}^{\frac{19}{18}} .
\end{aligned}
$$

That is, we have

$$
\int_{T_{k-1}}^{1}\left|\int_{\mathbb{R}^{3}} \frac{v_{k}}{|u|} u \nabla P_{k 2} d x\right| d t \leqslant C 2^{\frac{k}{9}} U_{k-1}^{\frac{19}{18}} .
$$

So, by combining inequalities, we yield

$$
\begin{aligned}
U_{k} \leqslant 2^{\frac{7 k}{3}} C U_{k-1}^{\frac{5}{3}}+\int_{T_{k-1}}^{1}\left|\int_{\mathbb{R}^{3}} \frac{v_{k}}{|u|} u \nabla P d x\right| d t & \leqslant 2^{\frac{7 k}{3}} C U_{k-1}^{\frac{5}{3}}+C 2^{\frac{10 k}{9}} U_{k-1}^{\frac{19}{18}}+C 2^{\frac{k}{9}} U_{k-1}^{\frac{19}{18}} \\
& \leqslant 2^{\frac{7 k}{3}} C U_{k-1}^{\frac{19}{18}} .
\end{aligned}
$$

That is, we will have the result that

$$
U_{k} \leqslant C 2^{\frac{7 k}{3}} U_{k-1}^{\frac{19}{18}}
$$

for any $k \geqslant 1$.

6. Proof of Proposition 1.1. Now, we would like to establish proposition 1.1 on the foundation of proposition 1.2. To begin, let $C^{*}$ be the positive universal constant occuring in proposition 1.2. First, let show the proposition in the special case $\lambda=2$. We chose $T$ to be an arbritary chosen positive number greater than 2 , and let $u$ be a solution of the Navier-Stokes equation on $(0, \infty) \times \mathbb{R}^{3}$. In the case in which $u$ satisfies the condition that $\int_{0}^{T} \int_{\mathbb{R}^{3}}|u|^{6} d x d s \leqslant\left(C^{*}\right)^{6}$, we define the function $u^{*}$ by $u^{*}(s, x)=u(s+(T-1), x)$, which can be regarded to be another solution of the Navier-Stokes equation on $[-1,1] \times \mathbb{R}^{3}$ satsifying

$$
\int_{-1}^{1} \int_{\mathbb{R}^{3}}\left|u^{*}\right|^{6} d x d s=\int_{T-2}^{T} \int_{\mathbb{R}^{3}}|u|^{6} d x d s \leqslant \int_{0}^{T} \int_{\mathbb{R}^{3}}|u|^{6} d x d s \leqslant\left(C^{*}\right)^{6} .
$$

Hence, we have $\left\|u^{*}\right\|_{L^{6}\left([-1,1] \times \mathbb{R}^{3}\right)} \leqslant C^{*}$. So, it follows from the conclusion of proposition 1.2 that $\|u(T, \cdot)\|_{L^{\infty}\left(\mathbb{R}^{3}\right)}=\left\|u^{*}(1, \cdot)\right\|_{L^{\infty}\left(\mathbb{R}^{3}\right)} \leqslant 1$.

So, the above argument shows that

- we have $\|u(T, \cdot)\|_{L^{\infty}\left(\mathbb{R}^{3}\right)} \leqslant 1$, if $T>2$, and $u$ is a solution of the Navier-Stokes equation satisfying $\int_{0}^{T} \int_{\mathbb{R}^{3}}|u|^{6} d x d s \leqslant\left(C^{*}\right)^{6}$. 
Next, we also need to deal with the case in which the solution $u$ satisfies the condition that $\int_{0}^{T} \int_{\mathbb{R}^{3}}|u|^{6} d x d s>\left(C^{*}\right)^{6}$. In this case, let us consider the function $u_{\varepsilon}$ defined by $u_{\varepsilon}(t, x)=\varepsilon u\left(\varepsilon^{2} t, \varepsilon x\right)$, in which $\varepsilon>0$ is arbritary. Then, by applying the change of variable formula, it is easy to see that

$$
\int_{0}^{\frac{T}{\varepsilon^{2}}} \int_{\mathbb{R}^{3}}\left|u_{\varepsilon}\right|^{6} d x d s=\varepsilon \int_{0}^{T} \int_{\mathbb{R}^{3}}|u|^{6} d x d s .
$$

So, by taking $\varepsilon=\left(C^{*}\right)^{6} \cdot\left\{2 \int_{0}^{T} \int_{\mathbb{R}^{3}}|u|^{6} d x d s\right\}^{-1}$, we yield

$$
\int_{0}^{\frac{T}{\varepsilon^{2}}} \int_{\mathbb{R}^{3}}\left|u_{\varepsilon}\right|^{6} d x d s=\frac{\left(C^{*}\right)^{6}}{2}<\left(C^{*}\right)^{6} .
$$

The last inequality signifies that the solution $u_{\varepsilon}$ falls back to the first case in this discussion. Hence, it follows directly form the conclusion we made for the frist case that $u_{\varepsilon}$ must satisfies $\left\|u_{\varepsilon}\left(\frac{T}{\varepsilon^{2}}, \cdot\right)\right\|_{L^{\infty}\left(\mathbb{R}^{3}\right)} \leqslant 1$. So, we eventually have

$$
\|u(T, \cdot)\|_{L^{\infty}\left(\mathbb{R}^{3}\right)}=\frac{1}{\varepsilon}\left\|u_{\varepsilon}\left(\frac{T}{\varepsilon^{2}}, \cdot\right)\right\|_{L^{\infty}\left(\mathbb{R}^{3}\right)} \leqslant \frac{1}{\varepsilon}=\frac{2}{\left(C^{*}\right)^{6}} \int_{0}^{T} \int_{\mathbb{R}^{3}}|u|^{6} d x d s .
$$

As a result, by all the discussion we made as above, we conclude that, no matter in which case, we always have the following inequality to be valid for any $T>2$, and any solution $u$ of the Navier-Stokes equation on $(0, \infty) \times \mathbb{R}^{3}$

$$
\|u(T, \cdot)\|_{L^{\infty}\left(\mathbb{R}^{3}\right)} \leqslant A\left\{1+\int_{0}^{T} \int_{\mathbb{R}^{3}}|u|^{6} d x d s\right\},
$$

where $A$ is the universal constant defined by $A=\max \left\{1, \frac{2}{\left(C^{*}\right)^{6}}\right\}$. This gives the proof of Proposition 1.1 in the special case $\lambda=2$.

Next, let $\lambda$ be a fixed positive number satisfying $0<\lambda<2$. As usual, let $u$ be a solution of the Navier-Stokes equation on $(0, \infty) \times \mathbb{R}^{3}$. Now, let us consider the function $w$ which is defined by

$$
w(t, x)=\left(\frac{\lambda}{2}\right)^{\frac{1}{2}} u\left(\frac{\lambda}{2} t,\left(\frac{\lambda}{2}\right)^{\frac{1}{2}} x\right) .
$$

Then, by applying the above case to $w$, we have the following estimation, which is valid for any $T>\lambda$.

$$
\begin{aligned}
\|u(T, \cdot)\|_{L^{\infty}\left(\mathbb{R}^{3}\right)} & \leqslant\left(\frac{2}{\lambda}\right)^{\frac{1}{2}}\left\|w\left(\frac{2 T}{\lambda}, \cdot\right)\right\|_{L^{\infty}\left(\mathbb{R}^{3}\right)} \\
& \leqslant\left(\frac{2}{\lambda}\right)^{\frac{1}{2}} A\left\{1+\int_{0}^{\frac{2 T}{\lambda}} \int_{\mathbb{R}^{3}}|w|^{6} d x d s\right\} \\
& \leqslant\left(\frac{2}{\lambda}\right)^{\frac{1}{2}} A\left\{1+\left(\frac{\lambda}{2}\right)^{\frac{1}{2}} \int_{0}^{T} \int_{\mathbb{R}^{3}}|u|^{6} d x d s\right\} \\
& \leqslant\left(\frac{2}{\lambda}\right)^{\frac{1}{2}} A\left\{1+\int_{0}^{T} \int_{\mathbb{R}^{3}}|u|^{6} d x d s\right\} .
\end{aligned}
$$
$\left(\frac{2}{\lambda}\right)^{\frac{1}{2}} A$.

This gives proposition 1.1, where the universal constant $A_{\lambda}$ is chosen to be $A_{\lambda}=$ 
7. Establishment of Theorem 1. Finally, we are now ready to establish the conclusion of Theorem 1 on the foundation of proposition 1.1. We make use of the following result due to Kato [12] (see also the book of Lemarié-Rieusset [14]).

Theorem 2. Let $p>3$. Then, for any given initial datum $u_{0} \in L^{p}\left(\mathbb{R}^{3}\right)$ satisfying $\operatorname{div}\left(u_{0}\right)=0$, there exists a positive $T^{*}$ and a unique weak solution $u \in$ $C\left(\left[0, T^{*}\right) ; L^{p}\left(\mathbb{R}^{3}\right)\right)$ for the Navier-Stokes equation on $\left(0, T^{*}\right) \times \mathbb{R}^{3}$ so that $u(0, \cdot)=u_{0}$. This solution is then smooth on $\left(0, T^{*}\right) \times \mathbb{R}^{3}$. In addition, such a unique solution will also satisfies the extra condition that $u(t, \cdot) \in C_{0}\left(\mathbb{R}^{3}\right)$, for all $t \in\left(0, T^{*}\right)$.

To begin, let $u$ be a weak solution of the Navier-Stokes equation on $(0, \infty) \times$ $\mathbb{R}^{3}$ satisfying the condition that $\int_{0}^{\infty} \int_{\mathbb{R}^{3}} \frac{|u|^{5}}{\log (1+|u|)} d x d s<\infty$. Then, by using the elementary inequality $\log (1+t) \leqslant t$, which is valid for all $t \geqslant 0$, we can deduce at once that

$$
\int_{0}^{\infty} \int_{\mathbb{R}^{3}}|u|^{4} d x d s \leqslant \int_{0}^{\infty} \int_{\mathbb{R}^{3}} \frac{|u|^{5}}{\log (1+|u|)} d x d s<\infty
$$

Now, let $\lambda \in(0,2)$ to be arbritary chosen and fixed. Since $\int_{0}^{\infty} \int_{\mathbb{R}^{3}}|u|^{4} d x d s<\infty$, it follows that the quantity $\int_{\mathbb{R}^{3}}|u(t, x)|^{4} d x$ must be finite for almost every $t \in(0, \infty)$. So, with respect to $\lambda$, we can choose some $\tau_{0}$ with $0<\tau_{0}<\lambda$ in such a way that $\int_{\mathbb{R}^{3}}\left|u\left(\tau_{0}, x\right)\right|^{4} d x<\infty$, or equivalently $u\left(\tau_{0}, \cdot\right) \in L^{4}\left(\mathbb{R}^{3}\right)$. So, by using a simple shifting technique, we may apply the Kato's Theorem quoted as above to deduce that there exists some positive constant $T^{*}>\tau_{0}$ so that our weak solution $u$ is smooth on $\left(\tau_{0}, T^{*}\right) \times \mathbb{R}^{3}$. Now, let us choose such a $T^{*}$ to be the largest one for which $u$ is smooth on $\left(\tau_{0}, T^{*}\right) \times \mathbb{R}^{3}$, and we would like to argue that $T^{*}=+\infty$. To this end, let us assume on the contray that $T^{*}<+\infty$, and we will derive a contradication by showing that $u \in L^{\infty}\left(\left[\tau, T^{*}\right] \times \mathbb{R}^{3}\right)$ is valid for some $\tau \in\left(\tau_{0}, T^{*}\right)$ slightly bigger than $\tau_{0}$.

For some technical purpose, we would like to pick up two numbers $\tau_{1}$ and $\tau_{2}$ which verify the condition that $\tau_{0}<\tau_{1}<\tau_{2}<\min \left\{\lambda, T^{*}\right\}$. Once $\tau_{1}$ and $\tau_{2}$ are chosen, they will be fixed. Now, from our original weak solution $u$, we can construct another solution $v$ by requiring that $v(t, x)=u\left(t+\tau_{1}, x\right)$. Now, by applying the conclusion of proposition 1.1 to the weak solution $v$ and the number $\tau_{2}-\tau_{1}$, we can at once deduce that we have the following inequality

$$
\|v(t, \cdot)\|_{L^{\infty}\left(\mathbb{R}^{3}\right)} \leqslant A\left\{1+\int_{0}^{t} \int_{\mathbb{R}^{3}}|v|^{6} d x d s\right\}
$$

to be valid for all $t \in\left(\tau_{2}-\tau_{1}, T^{*}-\tau_{1}\right)$, in which $A$ is some universal constant depending only on $\tau_{2}-\tau_{1}$. However, this means the same as saying that we have the following inequality

$$
\left\|u\left(t+\tau_{1}, \cdot\right)\right\|_{L^{\infty}\left(\mathbb{R}^{3}\right)} \leqslant A\left\{1+\int_{\tau_{1}}^{t+\tau_{1}} \int_{\mathbb{R}^{3}}|u|^{6} d x d s\right\}
$$

which is valid for all $t \in\left(\tau_{2}-\tau_{1}, T^{*}-\tau_{1}\right)$. Hence, it follows that we can make the following conclusion

- for every $t \in\left(\tau_{2}, T^{*}\right)$, we have $\|u(t, \cdot)\|_{L^{\infty}\left(\mathbb{R}^{3}\right)} \leqslant A\left\{1+\int_{\tau_{1}}^{t} \int_{\mathbb{R}^{3}}|u|^{6} d x d s\right\}$, in which $A$ is some universal constant depending only on $\tau_{2}-\tau_{1}$. 
At this stage, we are ready to apply the Gronwall's argument in the theory of ordinary differential equations as follow. For this purpose, we take $\psi(t)=t \cdot \log (1+t)$, which is a strictly increasing positive valued function on $(0, \infty)$ satisfying the condition that

$$
\int_{1}^{\infty} \frac{1}{\psi(t)} d t=\infty
$$

Then, it follows from our last inequality that

$$
\begin{aligned}
\|u(t, \cdot)\|_{L^{\infty}\left(\mathbb{R}^{3}\right)} & \leqslant A\left\{1+\int_{\tau_{1}}^{t} \int_{\mathbb{R}^{3}} \psi(|u|) \frac{|u|^{5}}{\log (1+|u|)} d x d s\right\} \\
& \leqslant A\left\{1+\int_{\tau_{1}}^{t} \psi\left(\|u\|_{L^{\infty}\left(\mathbb{R}^{3}\right)}\right) \int_{\mathbb{R}^{3}} \frac{|u|^{5}}{\log (1+|u|)} d x d s\right\},
\end{aligned}
$$

which is valid for all $t \in\left(\tau_{2}, T^{*}\right)$.

Next, we put $F(t)=\|u(t, \cdot)\|_{L^{\infty}\left(\mathbb{R}^{3}\right)}$. Then, the above inequality can be rewritten as

$$
F(t) \leqslant A\left\{1+\int_{\tau_{1}}^{t} \psi(F(s)) G(s) d s\right\}
$$

for all $t \in\left(\tau_{2}, T^{*}\right)$, where $G$ is the function defined by $G(s)=\int_{\mathbb{R}^{3}} \frac{|u|^{5}}{\log (1+|u|)} d x$. Furthermore, we notice that by the hypothesis of Theorem 1, the function $G$ must satisfies the condition that

$$
\int_{0}^{\infty} G(s) d s=\int_{0}^{\infty} \int_{\mathbb{R}^{3}} \frac{|u|^{5}}{\log (1+|u|)} d x d s<\infty .
$$

Here, for the sake of convenience, we define

$$
H(t)=A\left\{1+\int_{\tau_{1}}^{t} \psi(F(s)) G(s) d s\right\}
$$

for all $t \in\left(\tau_{1}, T^{*}\right)$. Then, our last inequality can be rewritten as

- $F(t) \leqslant H(t)$, for all $t \in\left(\tau_{2}, T^{*}\right)$.

Since $\psi$ is a strictly increasing positive valued function on $(0, \infty)$, it follows at once that

$$
\frac{d H}{d t}=A \psi(F(t)) G(t) \leqslant A \psi(H(t)) G(t),
$$

which is valid for all $t \in\left(\tau_{2}, T^{*}\right)$. That is, we have the fact that

- for every $t \in\left(\tau_{2}, T^{*}\right)$, we have $\frac{d H}{d t} \leqslant A \psi(H(t)) G(t)$.

As a result, by taking integration in time over the interval $\left(\tau_{2}, t\right)$, for $\tau_{2}<t<T^{*}$, it follows at once that 


$$
\Psi(H(t))-\Psi\left(H\left(\tau_{2}\right)\right) \leqslant A \int_{\tau_{2}}^{t} G(s) d s
$$

for all $t \in\left(\tau_{2}, T^{*}\right)$, in which $\Psi$ is the function defined by $\Psi(y)=\int_{A}^{y} \frac{1}{\psi(y)} d y$. Hence, we can deduce that

$$
\text { - } \sup _{t \in\left(\tau_{2}, T^{*}\right)} \Psi(H(t)) \leqslant \Psi\left(H\left(\tau_{2}\right)\right)+A \int_{\tau_{2}}^{T^{*}} G(s) d s .
$$

At this stage, in order to complete the Gronwall's argument successfully, we definitely need to show that $H\left(\tau_{2}\right)$ is finite. To achive this, let us recall that we have already used the Kato's Theorem to deduce that our original weak solution $u$ must satisfies $u \in L_{l o c}^{\infty}\left(\tau_{0}, T^{*} ; L^{\infty}\left(\mathbb{R}^{3}\right)\right)$, and this at once tells us that $\|u\|_{L^{\infty}\left(\left[\tau_{1}, \tau_{2}\right] \times \mathbb{R}^{3}\right)}=$ $\sup _{t \in\left[\tau_{1}, \tau_{2}\right]} F(t)<+\infty$, because of the fact that $0<\tau_{0}<\tau_{1}<\tau_{2}<\min \left\{\lambda, T^{*}\right\}$. Hence, it follows immediately that

$$
H\left(\tau_{2}\right) \leqslant A\left\{1+\psi\left(\|u\|_{L^{\infty}\left(\left[\tau_{1}, \tau_{2}\right] \times \mathbb{R}^{3}\right)}\right) \int_{\tau_{1}}^{\tau_{2}} G(s) d s\right\}<+\infty
$$

So, we can now combine $H\left(\tau_{2}\right)<\infty$, and $\int_{0}^{\infty} G(s) d s<\infty$ to deduce that

$$
\text { - } \sup _{t \in\left(\tau_{2}, T^{*}\right)} \Psi(H(t)) \leqslant \Psi\left(H\left(\tau_{2}\right)\right)+\int_{\tau_{2}}^{T^{*}} G(s) d s<\infty .
$$

Since $\int_{A}^{+\infty} \frac{1}{\psi(y)} d y=+\infty$, this will force us to admit that $\sup _{t \in\left(\tau_{2}, T^{*}\right)} H(t)<\infty$. Hence, we eventually have the conclusion that

$$
\text { - } \sup _{t \in\left(\tau_{2}, T^{*}\right)}\|u(t, \cdot)\|_{L^{\infty}\left(\mathbb{R}^{3}\right)} \leqslant \sup _{t \in\left(\tau_{2}, T^{*}\right)} H(t)<\infty .
$$

Since our weak solution $u$ now satisfies the condition $u \in L^{\infty}\left(\tau_{2}, T^{*} ; L^{\infty}\left(\mathbb{R}^{3}\right)\right)$, we already know that the assumption $T^{*}<+\infty$ cannot be valid since, by the standard Prodi-Serrin argument, our Leray Hopf weak solution $u$ must blow up in $L^{\infty}$ at $T^{*}$. So, we can conclude that $u$ is smooth on $\left(\tau_{2}, \infty\right) \times \mathbb{R}^{3}$, and hence we have the conclusion that $u$ is smooth on $(\lambda, \infty) \times \mathbb{R}^{3}$ (notice that $\tau_{2}<\lambda$ ). Since $\lambda \in(0,2)$ is arbritary chosen in the above argument, we can finally deduce that any weak solution $u$ satisfying the hypothesis of Theorem 1 must be smooth on $(0, \infty) \times \mathbb{R}^{3}$.

\section{REFERENCES}

[1] J. T. Beale, T. Kato, And A. Majda, Remarks on the breakdown of smooth solutions for the 3-D Euler equations, Comm. Math. Phys., 94 (1984), pp. 61-66.

[2] H. Beirão Da Veiga, A new regularity class for the Navier-Stokes equations in $\mathbf{R}^{n}$, Chinese Ann. Math. Ser. B, 16 (1995), pp. 407-412. A Chinese summary appears in Chinese Ann. Math. Ser. A, 16 (1995), p. 797.

[3] H. BEIRÃO DA VEIGA, Vorticity and regularity for solutions of initial-boundary value problems for the Navier-Stokes equations, Hyperbolic problems and regularity questions, pp. 39-47, Trends Math, Birkhauser, Basel, 2007.

[4] H. Beirão da Veiga and L.C. Berselli. Vorticity and regularity for flows under the Navier boundary condition. Commun. Pure Appl. Anal. 5(2006), no. 4, 907-918.

[5] H. BEIRÃO DA VeIGA, Vorticity and regularity for viscous incompressible flows under the Dirichlet boundary condition. Results and related open problems, Journal of Math. Fluid Mech., 9 (2007), pp. 506-516.

[6] H. Beirão Da Veiga and L.C. Berselli, Green's matrices, vorticity direction, and regularity up to the boundary, to appear in J. Diff. Equations. 
[7] P. Constantin and Ch. Fefferman, Direction of vorticity and the problem of global regularity for the Navier-Stokes equations, Indiana Univ. Math. J., 42 (1993), pp. 775-789.

[8] E. De Giongi, Sulla differenziabilità e l'analiticità delle estremali degli integrali multipli regolari, Mem. Accad. Sci. Torino. Cl. Sci. Fis. Mat. Nat. (3), 3 (1957), pp. 25-43.

[9] C. HE, Regularity for solutions to the Navier-Stokes equations with one velocity component regular, Electron. J. Differential Equations, (2002), pp. 1-13.

[10] E. Hopf, Über die Anfangswertaufgabe für die hydrodynamischen Grundgleichungen, Math. Nachr., 4 (1951), pp. 213-231.

[11] L. Iskauriaza, G. A. Serëgin, And V. Shverak, $L_{3, \infty}$-solutions of Navier-Stokes equations and backward uniqueness, Uspekhi Mat. Nauk, 58 (2003), pp. 344.

[12] T. KATO, Strong $L^{p}$-solutions of the Navier-Stokes equation in $\mathbf{R}^{m}$, with applications to weak solutions, Math. Z., 187 (1984), pp. 471-480.

[13] H. Kozono and Yasushi TANiuchi, Bilinear estimates in BMO and the Navier-Stokes equations, Math. Z., 235 (2000), pp. 173-194.

[14] P. G. LemariÉ-RIeusset, Recent developments in the Navier-Stokes problem, volume 431 of "Chapman \& Hall/CRC Research Notes in Mathematics", Chapman \& Hall/CRC, Boca Raton, FL, 2002.

[15] J. LeRAY, Sur le mouvement d'un liquide visqueux emplissant l'espace, Acta. Math., 63 (1934), pp. $183-248$.

[16] S. MontgomerY-Smith, Conditions implying regularity of the three dimensional Navier-Stokes equation, Appl. Math., 50 (2005), pp. 451-464.

[17] P. Penel and M. Pokorný. Some new regularity criteria for the Navier-Stokes equations containing gradient of the velocity. Appl. Math., 49(5):483-493, 2004.

[18] G. Prodi, Un teorema di unicità per le equazioni di Navier-Stokes, Ann. Mat. Pura Appl. (4), 48 (1959), pp. 173-182.

[19] G. Seregin And V. Šverák, Navier-Stokes equations with lower bounds on the pressure, Arch. Ration. Mech. Anal., 163 (2002), pp. 65-86.

[20] J. SERrin, The initial value problem for the Navier-Stokes equations, In "Nonlinear Problems (Proc. Sympos., Madison, Wis.", pp. 69-98, Univ. of Wisconsin Press, Madison, Wis., 1963.

[21] M. Struwe, On partial regularity results for the Navier-Stokes equations, Comm. Pure Appl. Math., 41 (1988), pp. 437-458.

[22] A. VASSEUR, A new proof of partial regularity of solutions to Navier-Stokes equations, to appear in NoDEA.

[23] Y. ZHOU, A new regularity criterion for the Navier-Stokes equations in terms of the gradient of one velocity component, Methods Appl. Anal., 9 (2002), pp. 563-578.

[24] Y. ZHOU, Direction of vorticity and a new regularity criterion for the Navier-Stokes equations, ANZIAM J., 46 (2005), pp. 309-316.

[25] Y. ZHOU, A new regularity criterion for the Navier-stokes equations in terms of the direction of vorticity, Monatsh Math., 144 (2005), pp. 251-257.

[26] Y. ZHou, A new regularity criterion for weak solutions to the Navier-Stokes equations, J. Math. Pures Appl. (9), 84 (2005), pp. 1496-1514. 